\title{
Civilisations
}

Revue internationale d'anthropologie et de sciences

humaines

56 | 2007

Après la catastrophe

\section{Le jour où les mondes disparurent}

\section{Anne-Marie Vuillemenot}

\section{OpenEdition}

\section{Journals}

Édition électronique

URL : http://journals.openedition.org/civilisations/136

DOI : $10.4000 /$ civilisations. 136

ISSN : 2032-0442

\section{Éditeur}

Institut de sociologie de l'Université Libre de Bruxelles

\section{Édition imprimée}

Date de publication : 1 décembre 2007

Pagination : 87-101

ISBN : 2-87263-016-3

ISSN : 0009-8140

\section{Référence électronique}

Anne-Marie Vuillemenot, « Le jour où les mondes disparurent », Civilisations [En ligne], 56 | 2007, mis en ligne le 01 décembre 2010, consulté le 13 novembre 2019. URL : http://journals.openedition.org/ civilisations/136; DOI : 10.4000/civilisations. 136 


\title{
Le jour où les mondes disparurent
}

\author{
Anne-Marie VUILLEMENOT
}

Résumé : Cet article s'interroge sur la ré-humanisation du monde postmoderne kazakh à partir des ruptures, des pertes et des disparitions qui ont émaillé la vie de familles de bergers semi-nomades de la soviétisation à nos jours. Le radicalement nouveau, le tout autre et le tout autrement s'avèrent-ils insaisissables pour les bergers kazakhs à travers le prisme de leurs représentations traditionnelles des mondes? Leurs repères spatiotemporels s'en trouvent-ils bouleversés au point de ruiner leurs anciens rapports aux mondes?

Mots-clés : Kazakhstan, semi-nomades, catastrophes, vie quotidienne.

Summary: This paper deals with the re-humanisation of the Kazak post-modern world through the ruptures, the losses and the disappearances which have surrounded the family lives of semi-nomad shepherds from the era of sovietisation to the present day. Can we consider the radically new, the totally different and the all otherly to be ungraspable for the Kazak sheperds through the prism of their traditional representations of the worlds? Are their landmarks in space and time disturbed to the point of ruining their old relation to the worlds?

Key words: Kazakhstan, seminomads, catastrophies, everyday life. 
7 he day the world collapsed est une nouvelle kazakhe qui relate une journée de printemps dans les années soixante, lorsque l'ex-URSS décida d'engager des essais nucléaires à ciel ouvert en déplaçant une partie de la population des bergers des steppes et en laissant l'autre sur place afin de pouvoir observer un échantillon témoin d'humains irradiés. L'herbe s'est mise à pousser étrangement, les moutons à perdre leur toison et les humains, les djigits, nobles descendants des cavaliers des steppes, à tomber gravement malades. Le choc indescriptible de cet énorme champignon s'élevant au dessus de l'immensité semi désertique a marqué et marque encore des générations du sceau de l'uranium enrichi. Cette nouvelle publiée en anglais, ne fut accessible qu'à un petit nombre de lecteurs locaux.

C'est malheureusement sur ce fond de poubelle nucléaire de la grande Russie soviétique qu'il faut imaginer la beauté folle des steppes et des montagnes kazakhes. La radioactivité n'abîme pas visuellement le paysage, c'est formidable ! Partant, très peu de personnes surent ce qui se tramait dans la steppe. Ce n'est qu'après l'indépendance et à l'initiative du mouvement d'opposition Nevada-Semi ${ }^{1}$ que la question de la pollution nucléaire fut ouvertement posée.

Bien avant ce cataclysme, plusieurs fractures émaillèrent la vie des familles kazakhes de bergers avec lesquelles je travaille depuis 1992. A travers leur récit, cet article interroge les transformations de l'être au monde kazakh, certaines liées aux événements extérieurs, d'autres à ces facteurs internes de changement qui existent dans toutes les sociétés humaines. Comme l'écrit Peter Sloterdijk (2002 : 26), « depuis que les temps sont devenus 'modernes', au sens précis du terme, l'être-dans-le-monde signifie devoir s'agripper à l'écorce terrestre et implorer la pesanteur - au-delà du giron et de l'enveloppe ».

Aussi, s'agit-il de se pencher sur la ré-humanisation du monde postmoderne kazakh et pas seulement de souligner l'irrémédiable des ruptures, des pertes et des disparitions. Le radicalement nouveau, le tout autre et le tout autrement s'avèrent-ils insaisissables pour les bergers kazakhs à travers le prisme de leurs représentations traditionnelles des mondes ? Leurs repères spatio-temporels s'en trouvent-ils bouleversés au point de ruiner leurs anciens rapports aux mondes?

\section{Quelques repères historiques}

$\mathrm{Au}$ cœur de l'Eurasie, l'Asie centrale a subi les influences indienne, chinoise, grecque, mongole, turque, arabe et russe. De même, ces régions ont connu les empires d'Alexandre, de Gengis Khan, de Tamerlan (Timour), de la Russie tsariste et enfin soviétique. La population kazakhstanaise ${ }^{2}$ contemporaine se présente ainsi comme une mosaïque d'origines, de langues, de religions.

1 Ce parti écologiste dirigé par l'écrivain Oljaz Sulomeinov, fut fondé en 1991 et dissout en 1994. Son chef de file fut "envoyé » en Italie comme premier ambassadeur du Kazakhstan. Le choix du nom NevadaSemi soulignait la similarité des situations : expériences nucléaires américaines au Nevada et expériences nucléaires soviétiques dans la région de Semipalatin. Dans les deux cas, des populations témoins devaient servir à mesurer différents effets de la radioactivité sur les humains.

2 Différencier la société kazakhe de la société kazakhstanaise (qui comprend de nombreuses populations non kazakhes) pose certainement problème dans une logique d'Etat-nation. Il en est tout autrement pour les populations locales qui ont conservé la séparation soviétique de nationalité/citoyenneté. 
Les langues turques ont emprunté au mongol et au persan, puis au russe, un grand nombre de mots. A Almaty, on parle : russe, kazakh, ouzbek, kirghiz, turc, tchéchtène, tadjik, turkmen, allemand ( $c f$. les populations de la Volga déplacées par Staline), polonais, grec... mais aussi anglais, la langue du nouvel âge marchand.

Les différentes strates du paysage ${ }^{3}$ religieux comprennent le chamanisme, la religion zoroastrienne, la chrétienté nestorienne, le bouddhisme, l'hindouisme, le soufisme et différentes sortes d'islam populaire, l'orthodoxie russe et, aujourd'hui, un islam plus intégriste ainsi que la chrétienté des églises charismatiques et pentecôtistes.

La société kazakhe traditionnelle révèle une grande complexité dans son organisation et suscite une série de controverses quant à son origine.

Le nom des Kazakhs est accolé à celui des Kirghizs pendant des siècles, l'ensemble des tribus se répartissant sur des territoires allant du sud de la Sibérie au nord de l'Afghanistan, de l'ouest de la Chine aux rives est de la Caspienne. C'est à partir du $16^{\mathrm{e}}$ siècle que différentes sources signalent les Kazakhs et livrent des informations sur leur mode de vie. Les siècles précédents ont vu la naissance, l'apogée et l'effondrement d'empires construits par des nomades, qui prennent le pas sur des civilisations de sédentaires et qui se font peu à peu phagocyter par elles. Des noms mythiques se succèdent: les Scythes (13 ${ }^{\mathrm{e}}$ siècle avant J.-C.), les Sarmates ( $4^{\mathrm{e}}$ siècle avant J.-C.), les Huns (4e et $5^{\mathrm{e}}$ siècles), puis les empires turco-mongols qui s'opposent à l'empire chinois, et dont Gengis Khan $\left(12^{\mathrm{e}}-13^{\mathrm{e}}\right.$ siècles) incarne encore aujourd'hui l'une des figures les plus glorieuses ${ }^{4}$.

Pendant l'essentiel de leur histoire, les Kazakhs, quel que soit le nom qu'on leur donnait à l'époque, ne furent pas organisés en nation, mais en tribus dispersées ou incluses dans une fédération provisoire de tribus. Les premières organisations « unies » kazakhes apparaissent en 1698 sous la direction du Khan Tauke. La division par les Kazakhs de leur nation en trois $z h u z^{5}$ (uli (aînée), orta (moyenne) et keche (cadette)) ${ }^{6}$, comme le présente la structure sociale contemporaine, n'apparaît qu'au $18^{\mathrm{e}}$ siècle. La zhuz rassemble un certain nombre de tribus, nombre variable suivant les époques et les alliances. A chaque zhuz correspond une fonction particulière. La zhuz cadette occupe traditionnellement le pouvoir, c'est d'elle que sont issus les khans. La zhuz moyenne rassemble les poètes, les écrivains et les artisans, la zhuz aînée est celle des bergers. En mettant au pouvoir un fils de berger issu de la zhuz aînée, au moment de l'indépendance, la Russie ex-soviétique s'est ainsi préservée d'un retour éventuel de la noblesse, retour d'autant plus hypothétique que des années de régime soviétique ont suffi pour éliminer la majorité de ceux qui formaient l'intelligentsia kazakhe.

3 J'emploie ce terme tel que le définit Arjun Apaduraï (2001).

4 Sur ces questions voir : Barthold (1962), Krader (1963), Dor (1975), Pelliot (1950), Valikhanov (18341860).

5 Le terme zhuz signifie littéralement le chiffre cent. Dans la steppe altaïque, le chiffre cent est lié à une organisation politico-militaire qui divise les armées en décurions, centurions, chiliards ... (Krader 1963 : 201). Zhuz est généralement traduit par « horde» dans les textes en russe, en anglais ou en français, terme qui n'est pas sans évoquer la « sauvagerie » ou la « barbarie » des cavaliers des steppes. En kazakh, la zhuz est parfois nommée aris (fleuve) et ouvre ainsi la géométrie des mondes kazakhs à la dimension horizontale ou encore à un puissant référent chamanique.

6 Traduction par l'auteur et littérale des termes employés en kazakh. 
Le dernier recensement de la population kazakhstanaise date de 1989, il différencie le pourcentage de chaque ethnie, mais pas celui de chaque $z h u z$ au sein de la population kazakhe. Cependant, même si cette ancienne structure ne se lit pas directement dans la vie quotidienne, elle continue à intervenir dans les réseaux de solidarité et dans le fonctionnement clientéliste du pouvoir en place.

La division en zhuz se doublait d'une organisation sociale en moitiés : les os blancs (la noblesse) et les os noirs (le peuple), division que les Kazakhs partageaient avec les Mongols. Aujourd'hui, d'autres facteurs sociaux organisent les groupes suivant deux axes : aîné/cadet, masculin/féminin. L'héritage soviétique complète cet aperçu de l'organisation sociale par une dichotomie marquée entre milieu urbain intensément russifié et milieu rural de culture kazakhe.

\section{Le monde selon Otinchi}

Lorsque, en 1993, j'interrogeai Otinchi, mon informateur principal, alors berger de son état, sur ce qu'était le monde pour lui, il signala d'abord l'arbre des mondes, l'axe vertical autour duquel les différents mondes s'organisent en étages superposés. Pour Otinchi, Dieu ${ }^{7}$ a sans doute créé toutes les formes de vie (si ce n'est pas lui, qui d'autre ? c'est donc lui par défaut), c'est pourquoi toutes doivent être respectées. L'humain, l'animal, le végétal et le minéral se partagent la planète et sont contraints à la recherche d'équilibres. Sur la création des mondes et du cosmos, Otinchi se fait plus discret : c'est encore Dieu, mais comment? Une certitude : chaque étoile est porteuse d' « âme », c'est-à-dire de principes vitaux ; en conséquence, le ciel s'observe avec précaution. En face de lui, le berger se fait craintif et respectueux, il ne voudrait en aucun cas attirer l'attention des djinns (figures d'esprits) sur lui et sa famille. Les interdits sont les interdits, peu importe la divinité ou l'esprit possesseur à qui ils se rapportent.

La vie est partout et en tout, la pierre vit, la montagne vit, la steppe vit. Pour Otinchi, quand un homme naît, des graines sont semées pour lui sur l'ensemble de la planète et sa vie terrestre se passe à récolter les fruits de ces graines. A la dernière récolte succède naturellement la mort qui entraîne l'homme dans « l'autre monde " ${ }^{8}$, où il vit comme sur terre avec sa famille, ses amis, sa yourte (hutte traditionnelle de feutre) et son bétail.

Dix ans plus tard, lors d'une discussion semblable autour d'un thé, Otinchi réaffirme sa croyance mais il ajoute que les mondes qui étaient les siens, hérités de ses parents et de ses ancêtres, ne sont plus les mondes de ses enfants ou de son petit-fils né alors que son grand-père avait déjà dû quitter sa yourte et son mode de vie semi-nomade. Aujourd'hui, Otinchi est chauffeur d'ambulance pour un hôpital situé en périphérie d'Ecik, petite bourgade accolée aux pieds des monts du Tien Shen, au sud-est du Kazakhstan. Malgré cette reconversion, Otinchi ne gagne plus de quoi nourrir sa famille. Sa femme et ses aînés assurent l'essentiel de la subsistance, ayant réussi à trouver un travail dans la ville d'Almaty. La famille s'en trouve dispersée mais Otinchi n'envisage pas de s'installer

7 Allah, Kodaï (dieu des Perses) ou Tengri (dieux-cieux des Turco-Mongols) apparaissent comme interchangeables sur cette aire culturelle.

8 Les morts et les ancêtres sont plurilocalisés dans toute l'aire turco-mongole. On consultera avec intérêt à ce sujet les travaux de Jean-Paul Roux (1984). 
en ville. Il conduit son ambulance comme il prenait soin de son cheval et parcourt les chemins comme il parcourait la steppe ou les pâturages de montagne pour secourir un voisin ou un ami. De la sorte, il se sent, au moins en partie, utile.

Que dois-je faire? me demande-il entre deux airs de dombra ${ }^{9}$, je ne veux pas vivre en ville. Nurjamal [sa femme], elle sait comment faire, moi pas. Au village, je peux encore parfois aller chasser en montagne. On part à deux avec Dakenaï [son ami berger, son bauir, c'est-à-dire son foie $\left.{ }^{10}\right]$ en contournant la route vers les pâturages de Saram Sarte. Tu sais la route aujourd'hui, ils l'ont fermée, elle est privée ; tu sais où on s'est rencontré la première fois pendant la transhumance, aujourd'hui c'est fermé. Ils ont construit des grandes datchas et on peut plus passer. T'imagines les moutons et les chevaux là? Plus rien, il ne reste plus rien, l'herbe pousse et personne pour la manger. Les loups descendent plus bas, ils ont faim. Nous on passe derrière, par l'autre vallée, on va se promener, chasser, on se souvient et puis on revient ». - Comment allez vous jusque-là ?, demandais-je.

- A cheval.

- Quels chevaux? Tu n'en as plus, Dakenaï non plus. »

- Il y a encore Askar Ara [l'aîné de l'ancien aoul (ensemble ou village de yourtes)], il a encore un peu de bétail qu'il garde au village et dans la journée, il monte les faire paître en montagne, là où c'est encore possible. Quand il peut, il nous passe deux chevaux et on part, un jour ou deux, j'ai jamais envie de redescendre; tu sais comment c'est là bas!

Le monde d'Otinchi et de bien d'autres était celui rythmé par les saisons et les changements de campements, celui de la vie dans la yourte avec la famille nucléaire et les voisins, celui en équilibre entre ciel et terre, là où doivent se tenir les humains. Ce « là bas » appartient désormais aux mondes inaccessibles et nourrit les récits que les plus anciens font aux jeunes générations nées sédentaires. Les grands espaces sans limites où le bétail se partage avec les loups même si toutes les nuits il faut surveiller et tirer au fusil, les tempêtes dévastatrices qui transforment parfois la montagne en torrents de boue ou encore, les rencontres insolites dans les steppes ou les montagnes, constituent autant de thèmes qui tiennent lieu et place aujourd'hui de récits des grandes épopées. L'histoire récente et ses bouleversements occupent une place de choix dans les discours lors de réunions familiales ou amicales. Les récits de vie s'inscrivent peu à peu dans l'imaginaire, rejoignant en cela la littérature et la poésie kazakhes.

Cependant, la reconstruction partielle de sa vie passée, même dans des montagnes et des steppes vides de yourtes, permet à Otinchi de s'inscrire dans son nouveau quotidien. Le passage des pâturages au village fut suffisamment déstabilisant pour l'empêcher de faire le saut du village à la ville comme ses enfants ou sa femme. "Qui conduira l'ambulance si je m'en vais? » dit-il. Les chauffeurs ne manquent pas, mais Otinchi a investi sa nouvelle fonction des référents de son ancienne vie. Sa nouvelle monture, sa voiture, lui permet de rester en contact avec les familles de bergers dans les différents villages, comme il le faisait en passant de yourte en yourte dans les pâturages. De plus, il réintègre son rôle de

$9 \quad$ Vielle traditionnelle à trois cordes.

10 Comme dans toute 1'aire turco-mongole, le terme foie désigne ici le siège des sentiments. Il se trouve aussi employé comme un terme de parenté qui signale pour l'homme, dans sa parentèle, ceux du même âge et qui assimile au lignage les amis considérés comme « vitaux » dans la vie quotidienne et le réseau d'entraide. 
chef de pâturage en servant d'intermédiaire entre le monde hospitalier, les médecins et les bergers se trouvant dans le besoin. Enfin, il se préserve une échappée : les escapades en montagne qui sont encore possibles dans cet environnement et impensables en ville ; par là même, il conserve un accès aux mondes d'en haut, ceux des divinités et des figures d'esprits qui habitent la montagne et n'ont plus personne avec qui converser puisque plus aucune fumée de foyer ne s'élève vers eux.

\section{De cataclysme en cataclysme}

L'histoire de cette famille, comme de tant d'autres, est ponctuée par les bouleversements de la grande Histoire, celle des Etats-nations, qui n'épargne personne. Il y eut d'abord l'arrivée des soviets, la collectivisation, puis le génocide d'un million et demi de Kazakhs entre 1927 et 1933. Les plus anciens s'en souviennent et racontent comment ceux qu'on envoyait coloniser l'empire soviétique creusaient des tranchées au moyen d'engins mécanisés et enterraient vivants des villages entiers, avant de piller les récoltes et de voler le bétail. D'autres se remémorent leur fuite vers la Chine et le Xinjiang dans le seul but de survivre, et, plus tard, la Révolution culturelle, les famines effroyables et la nécessité de rentrer en URSS, pour sauver sa vie une nouvelle fois. Et à leur retour, la découverte de la mécanisation, des kolkhozes et des sovkhozes, la survie vaille que vaille jusqu'à ce jour où l'immense champignon recouvrit la steppe. De pollution en pollution, l'environnement et les hommes ont résisté ; ils résistent encore. Dans ce tableau sinistre, le sort de la mer d'Aral ne représente jamais qu'un épisode parmi beaucoup d'autres.

L'indépendance en 1991 amène une nouvelle rupture, et avec elle, les bouleversements socioéconomiques de l'ouverture au grand marché international, le capitalisme sauvage et l'émergence des mafias. Le Kazakhstan, comme les autres républiques centreasiatiques, passe d'une autocratie de l'empire à une autocratie nationale. La privatisation des kolkhozes et des sovkhozes chasse les bergers de leurs yourtes et de leurs pâturages. Le bétail est abattu ou redistribué en quantité infime. Où nourrir son maigre cheptel alors qu'il n'est plus possible d'accéder aux pâturages ? Très vite, les bergers abattent leurs quelques bêtes pour vendre la viande au marché local. Derrière leurs étals de fortune, ces vendeurs improvisés peinent à écouler leur marchandise, connaissant à peine la valeur du kilogramme et faisant inévitablement chuter les prix, l'offre dépassant de loin la demande. Que faire au village ou en périphérie des grandes villes pour gagner sa vie ? La plupart des hommes ne connaissent d'autre vie que celle de berger. Ils sont capables de reconnaître les moindres murmures de la steppe ou les infimes changements de l'herbe de la montagne qui indiquent à quel moment il faut transhumer, quand la neige arrive et menace le troupeau. Ils sont familiers des loups, des serpents, des chacals ou des panthères des neiges, ils prennent soin de leurs chevaux comme d'eux-mêmes, savent comment faire croître et prospérer leur bétail, mais ils ne connaissent rien au milieu urbain et à la logique du marché du travail.

Leurs territoires s'étendaient à l'infini, entre ciel et terre, souvent de manière transfrontalière, car passer au Kirghizstan ou en Chine par les sentiers de montagne était un jeu d'enfants pour ces cavaliers émérites. Avant même de se trouver dépouillés de leurs compétences et de leur savoir en basculant dans un monde occidentalisé et technicisé, la première étape de leur déshumanisation commence par la perte du territoire. Dans une société où il est simplement indécent pour l'homme de passer sa journée dans la yourte, il est exclu que la maison ou l'appartement puisse borner leur vie. Mais où trouver un lieu 
lorsqu'aucun travail n'est disponible? Dans la rue? Dans les lieux publics? La vie au village ou en ville ne laisse pas de place aux hommes. Un certain nombre d'entre eux ont choisi le suicide plutôt que le non-lieu.

Alors que la vie dans les pâturages renvoyait l'homme à la steppe, à la montagne et plus généralement à l'extériorité, la femme s'inscrivait, elle, dans la yourte et dans tout ce qui symbolise l'intériorité. Depuis la sédentarisation, les femmes reconstruisent dans une pièce l'univers de la yourte et retrouvent en cela leurs repères et l'entretien du foyer. Cependant, nombre d'entre elles se trouvent contraintes de travailler au marché, de louer leurs services de cuisinières ou de ménagères afin d'assurer, trop souvent, le seul revenu de la famille. La rupture pour ces femmes se situe dans cette nécessité de travailler à l'extérieur pour faire vivre leur famille et dans le fait d'en devenir fréquemment l'unique pourvoyeur.

En avril 1994, je partage avec ces familles de bergers leurs dernières heures de nomadisme dans les pâturages de printemps dans la steppe, le long de l'Ili ; chacun se demande quelle attitude adopter. Les réunions se multiplient de yourte en yourte. C'est au cours de l'une d'elles que les femmes m'interpellent: "C'est facile pour toi de dire que nous devons défendre nos yourtes, tu viens, tu vis quelques temps avec nous et tu repars dans une maison où tu as de l'eau, du gaz, de l'électricité et la télévision ». Zina, qui vient de s'exprimer, rêve d'être au village pour suivre quotidiennement la série brésilienne «Prosta Maria » sur la chaîne de télévision kazakhstanaise. Il s'agit de l'histoire d'une pauvre paysanne engagée comme bonne en ville et qui connaît une ascension sociale fulgurante en devenant la directrice d'une agence de mannequins. Malgré le grand écart culturel et géographique entre le Brésil et le Kazakhstan, le principe d'identification est à l'œuvre et mobilise, là où il est accessible, de nombreuses personnes devant le petit écran. La rumeur de la steppe délivre avec retard mais fidèlement les épisodes que ne peuvent suivre les bergères dans la yourte. Les femmes révèlent en fait qu'elles aspirent aux commodités des villages ou des villes pour se libérer de l'intendance quotidienne dans la yourte. A distance, l'ailleurs urbain leur apparaît comme incroyablement plus attractif.

Le directeur kazakh du kolkhoze de Kaïnazar pour lequel travaillent tous ces bergers ne semble pas étranger à ces discussions qui débutent après sa dernière inspection. Il revient d'un voyage aux Pays-Bas où il a visité, dit-il, des "installations modernes et propres ». Il ne précise pas qu'il est en train de vendre le kolkhoze à une firme hollandaise de culture de pommes de terre et qu'il doit licencier tous les bergers le plus vite possible. Il se contente de persuader les bergers qu'ils vivent dans de très mauvaises conditions. En verve, il invente même un néologisme russe en expliquant que la vie dans la yourte est " anti sanitari ». Les blagues sur la situation d' " anti sanitari » se multiplient dans les jours qui suivent, mais l'idée fait son chemin et l'argument revient. "De toutes façons, notre vie ici n'est pas saine pour les enfants " commente une des bergères. Jusqu'alors, personne ne s'était jamais interrogé de la sorte.

Ces quelques échanges révèlent que les hommes ne sont pas prêts à quitter le nomadisme pastoral alors que les femmes rêvent d'urbanité ! En dernier ressort, les bergers n'auront pas à choisir puisque le choix a déjà était fait pour eux. Ils quitteront tous définitivement la yourte en mai 1994.

Bien des années après, Zina que je rencontre au village, me confie : " Anna, tu avais raison, en perdant notre yourte nous avons beaucoup perdu, mais à ce moment-là, nous ne savions pas ». En effet, face à ce bouleversement, la précarité et la paupérisation se 
sont accrues pour deux raisons : une baisse des revenus mais surtout une augmentation des besoins. De la yourte au village ou à la ville, la rupture se marque aussi en termes de consommation. La vie dans la steppe et l'ancien système de distribution kolkhozienne couvraient, en principe, les besoins des bergers. Il est vrai que ces derniers se contentaient de bien peu au regard de ce qu'offre aujourd'hui la société de marché. Le changement des modes de vie est spectaculaire et radical; on peut à cet égard parler d'un véritable changement de paradigme. Bien sûr, le mirage de l'urbanité et la découverte d'une tout autre réalité ne sont pas propres aux situations kazakhe et kazakhstanaise ; ils sont abondamment décrits dans une bonne partie des pays ex-soviétiques et appartiennent pleinement au champ des études migratoires qui montrent comment les problèmes économiques, sociaux et culturels liés à l'exode rural et à l'arrivée en ville amènent au désenchantement du milieu urbain. Dans son ouvrage Quand la misère chasse la pauvreté, Majid Rahnema (2003:18-19) montre très bien comment l'économicisation des sociétés induit une subordination croissante à l'économie et une prolifération des besoins - besoins qu'il est de plus en plus difficile, sinon impossible, de satisfaire, pour la majorité de la population. La situation contemporaine de ces bergers kazakhs correspond à ce schéma qui se trouve toutefois complexifié par deux autres facteurs : la recherche identitaire et l'individuation.

\section{Etre kazakh au $21^{\mathrm{e}}$ siècle}

Dans le Kazakhstan contemporain apparaissent en nombre des facteurs identitaires qui ne permettent pas de définir à proprement parler une nation kazakhe, mais qui montrent, suivant la définition de l'identité de Pierre Bonte et de Michel Izard, « une codification constamment renouvelée des différences culturelles entre groupes voisins » (Bonte et Izard 1991 : 243). Aussi les modes de construction, la transformation et la recomposition des normes sociales s'inscrivent-ils dans une dynamique qui dépasse de loin l'être-aumonde traditionnel de la personne kazakhe.

Les années '90 ont vu naître dans le discours officiel une volonté d'inscrire le Kazakhstan dans le concert des nations modernes. Pour ce faire, les référents au mode de vie nomade et aux ancêtres ont été instrumentalisés, laissant croire à la rencontre possible entre deux modes de vie radicalement différents. Une campagne intitulée « Kazakhstan 2030 » mettait en scène à la télévision kazakhe une voiture 4x4 se rendant dans les steppes sur fond de paysages grandioses évoquant la nature originelle. De ce véhicule descendaient des jeunes gens occidentalisés, vêtus à la dernière mode, qui rendaient visite à un vieil homme à la barbe blanche représentant la figure du sage : l'Aksakal. Cet aîné servait un thé dans la yourte et regardait repartir l'avenir de la nation, incarné par ces jeunes, non sans les avoir bénis. Entre la volonté officielle affichée et la réalité d'aujourd'hui, un gouffre s'est creusé. L'ensemble de la population kazakhstanaise (Kazakhs et autres) connaît aujourd'hui une situation tiers-mondiste : une minorité concentrant un maximum de richesses, les autres se trouvant réduits à la précarité. Dans sa volonté, soutenue internationalement, de construire un Etat-nation, le gouvernement kazakh s'appuie sur l'histoire et quelques grandes figures intellectuelles du passé, mais d'un passé soigneusement trié. On assiste ainsi à l'invention de lieux de mémoire et de pèlerinages sur des tombeaux de saints, de même qu'à l'apparition d'un artisanat qui réinstaure le port de vêtements traditionnels, faisant la joie des touristes. Des yourtes sont montées à chaque occasion de réjouissances, de fêtes nationales ou de commémorations 
de poètes et d'écrivains kazakhs. Et tout concourt à faire croire qu'il n'y a pas eu de rupture dans l'histoire de la nation.

Comme le soulignent Marlène Laruelle et Sébastien Peyrouse, ce vaste programme efface soigneusement les références au $20^{\mathrm{e}}$ siècle : "Dans ces reconstructions identitaires, les périodes coloniale et soviétique brillent avant tout par leur absence. La peur du contemporain anime en effet les pouvoirs centre-asiatiques, tous issus du régime précédent et qui préfèrent ignorer cette continuité, de peur de faire surgir leurs actuelles incohérences narratives » (Laruelle et Pérouse 2006 : 63).

Dans la volonté de construire une identité kazakhe, le facteur religieux se trouve instrumentalisé par le pouvoir en place ${ }^{11}$. Ce qui se joue dans le Kazakhstan contemporain me semble appartenir à un vaste ensemble de stratégies et de pratiques qui tendent à conjurer les effets du passage d'une économie assistée à un libéralisme effréné. A la recherche de solutions, la population kazakhe se tourne vers des systèmes de croyances qui s'alimentent aux grandes figures héroïques de l'Asie centrale des empires. L'enjeu est économique, mais aussi national, voire nationaliste. Dans ce cadre, la personne du bakhsi (chaman-soufi) apparaît comme un point de repère fiable alors que l'avenir est chargé d'incertitudes multiples. Origine ethnique et statut social confondus, nombreux sont ceux qui se rendent chez un bakhsi pour trouver une issue à leurs difficultés. En conséquence, les bakhsis se multiplient.

L'ensemble des pratiques religieuses contemporaines est soumis à deux types de déterminations : traditions d'une part, islam d'autre part. Mais de quelles traditions et de quel type d'islam s'agit-il ? L'aspect traditionnel fait référence à l'héritage préislamique du chamanisme sibérien, de la vieille religion zoroastrienne et des influences bouddhiste et nestorienne. L'islam populaire est presque exclusivement soufi. La période contemporaine se distingue par l'arrivée d'un islam plus normatif et de mouvements charismatiques tels que celui des pentecôtistes coréens.

Depuis 1992, je parcours les steppes et les montagnes kazakhes. J'ai eu l'occasion d'y rencontrer un grand nombre de bakhsis. De toutes mes enquêtes se dégage une constante : la présence du Coran dans les pratiques rituelles, au cours des consultations chez les bakhsis et dans les processus de guérison. Le livre s'y trouve systématiquement exposé, ouvert et touché mais jamais lu ! C'est dans ce contexte de bricolage rituel que les Kazakhs déclarent qu'ils sont musulmans. Même si Allah est évoqué, rien ne se fait explicitement en son nom. Il est dès lors très difficile de faire la part de l'influence de l'islam et celle du résultat des bouleversements sociaux culturels liés à la privatisation et à l'entrée dans la mondialisation.

Pour Serge Latouche, « la mégamachine globale rase tout ce qui dépasse du sol, mais elle enfonce les superstructures et conserve à son insu les fondations, en tout cas cette aspiration indéracinable : l'aspiration à une identité » (Latouche 2005 : 16). Cependant, les processus d'individuation et de recherche identitaire ne dépendent pas que d'influences externes globalisées, mais bien aussi de courants de pensées indigènes qui tendent à reconstruire l'être au monde kazakh contemporain. A cet égard, les travaux de chercheurs locaux apparaissent comme très contradictoires. D'aucuns, tel que Kamalbaï Mardanov (2004), défendent l'idée que le passé nomade est rayé de l'histoire collective à seule fin de conduire, de promesses creuses en promesses creuses, la population kazakhe et

11 Voir à ce sujet : Vuillemenot (2006). 
kazakhstanaise vers le mirage de la modernité. D'autres, tel que Abdumalik Nysanbayev (2004), parlent des perspectives offertes par une construction sociale rapprochant l'Orient de l'Occident, c'est-à-dire : alliant la «tradition » centre-asiatique à la mondialisation ambiante.

Mardanov signale que depuis plus ou moins 1995, de très profonds changements sont survenus dans la société kazakhe. Pour cet auteur, les jeunes générations ne sont pas instruites de l'histoire des ancêtres nomades et c'est un véritable plaidoyer pour le nomadisme qu'il nous livre :

Des milliers d'années d'histoire du nomadisme sont passées sous silence dans les manuels actuels, tout comme durant la période soviétique, ce qui influe négativement sur la formation de la conscience nationale. Comme en d'autres pays, les jeunes générations se tournent vers les villes. Chaque année les campagnes perdent de vingt-cinq mille à trente mille personnes ayant entre quinze et vingt-neuf ans. Dans les villages ne restent que les vieux, les infirmes et les indigents. Tous ceux qui le peuvent vont à Almaty ou Astana. Ils ne peuvent y trouver du travail, vivent à la périphérie dans des logements précaires. La nouvelle législation aura pour effet semble-t-il, de fixer pour toujours à leur « coin », la majorité de la population des villages. Le problème le plus urgent pour le gouvernement est la croissance rapide des villes et leur approvisionnement. Il est nécessaire de recourir au bon vieil élevage de pâturage, à l'image de ce qu'ont fait finalement les soviétiques et en se souvenant des usages des nomades. Pour répondre aux besoins des gens des villages et à ceux de la jeunesse, l'Etat répond par des slogans et par des promesses de tout résoudre en trois ans, ce à quoi les intéressés ne croient plus. Les problèmes des villages, de plus en plus brûlants d'année en année, ne sont pas un héritage du régime antérieur, c'est le pouvoir actuel qui en est le responsable ${ }^{12}$ (Mardanov 2004 : 436-437).

Pour Abdumalik Nysanbayev, les transformations sociales entamées depuis l'indépendance se présentent au contraire comme une véritable issue : "Since a new free Kazakh philosophy has been formed which has rejected the Marxist dogma of materialism and atheism, acknowledgement of the essence of a person, his spiritual soul and the ethical foundation of society should be considered as determinant» (Nysanbayev 2004 : 113). Cet auteur tente de prouver que la nouvelle vague philosophique kazakhe s'oriente vers un rapprochement Est-Ouest sur la base d'un " connais toi toi-même » contemporain, visant à proposer un programme d'enseignement axé sur les valeurs de la personne, de l'éthique, de l'amour, du respect de la nature, de l'harmonie et de la vertu ( $c f$. Nysanbayev 2004 : 111-112). Le surprenant de cet ouvrage réside dans le fait que seul l'accès à une individualité harmonieuse et équilibrée, construite sur un modèle non pas matérialiste, mais tout à la fois empreint de technologies contemporaines et de spiritualité bricolée à partir de différents courants, peut véritablement faire entrer le Kazakhstan et les Kazakhs dans la modernité ! Les facteurs communautaire et pluriethnique se trouvent totalement absents de ce projet. Comme si l'émergence d'un individu kazakhstanais permettait de gommer les problèmes rencontrés aujourd'hui par des populations dont les modes de vie s'inscrivaient jusqu'alors dans une logique communautaire (où la personne ne se concevait que prise dans un tissu familial et social et dans une obligation de dialogue avec 
les habitants des au-delà, les figures d'esprits et les divinités), populations qui font face aujourd'hui à des transformations constantes, très rapides et irréversibles. Si la seule issue proposée et défendue par les nouveaux philosophes kazakhs est de devenir l'Individu globalisé, comment les jeunes générations descendantes de bergers peuvent-elles se reconnaître dans la personne kazakhe « traditionnelle » riche de tous ses liens familiaux et sociaux et de ses principes vitaux (l'âme, le kyt et le souffle) ${ }^{13}$ ?

Parmi d'autres facteurs identitaires, la langue reste une question complexe au Kazakhstan. Il faut raisonner ici en termes de populations plus ou moins soviétisées et surtout différencier les zones urbaines des zones rurales. Le milieu urbain kazakh s'est trouvé intensément russifié et nombreux sont les Kazakhs des villes qui ont perdu complètement la langue kazakhe. Depuis l'indépendance, tout est fait pour inciter les Kazakhs à parler kazakh et à apprendre une langue épurée des idiomes russes qui s'y sont implantés depuis le $18^{\mathrm{e}}$ siècle.

Comme le montre H. Iankovski (2005), la posture de la langue kazakhe est et reste particulière. Dès 1990, une loi qui se présente d'emblée comme un objectif à atteindre pour l'an 2000, donne au kazakh le statut de langue d'Etat et conserve au russe le statut de langue officielle. Au cours des années '90, ce délai apparaît très vite comme non réaliste et un décret fixe à 2010 la réalisation de ce qui apparaît de plus en plus comme un vœu pieux et non comme une mesure efficace (Iankovski 2005 : 80). Alors que les médias n'arrivent pas à imposer le kazakh comme langue vernaculaire principale (par exemple, dans les émissions télévisées en kazakh, les publicités restent en russe), le kazakh est de plus en plus présent dans les productions écrites (idem : 81).

Aujourd'hui, l'apport massif, dû à l'exode rural, d'une population majoritairement kazakhophone en milieu urbain va-t-il changer la configuration linguistique des villes ? Rien n'est moins sûr. Le russe reste une langue transnationale au sein de l'ex-URSS et surtout, l'occidentalisation en cours donne à l'anglais un nouvel essor local.

\section{L'individuation, perte des repères collectifs}

La paupérisation et, parallèlement, l'augmentation des besoins ont réduit le réseau de solidarité traditionnel à une peau de chagrin. Jusqu'alors, dans les situations difficiles, l'appartenance ethnique n'intervenait pas. Dans le système traditionnel de croyances, il fallait s'entraider au nom de l'obligation faite aux humains de préserver l'équilibre des mondes. D'une certaine manière, la période soviétique avait cristallisé cet état de choses en offrant à tous un ennemi commun : le spectre de la répression. L'arrivée de la logique de marché a fait exploser cette référence. Tous les services de l'Etat sont devenus payants. L'eau, le téléphone, le gaz, l'électricité, le système de santé sont en voie de privatisation avec pour conséquence l'exclusion de ceux qui ne peuvent payer. Il s'ensuit non pas un développement des réseaux de solidarité, mais un repli sur soi.

Comment l'organisation cosmogonique des mondes a-t-elle pu disparaître aussi vite alors qu'elle avait résisté à la soviétisation ? Paradoxalement, l'Homo sovieticus permettait

13 La personne kazakhe reçoit à la naissance trois principes vitaux essentiels : une âme individuelle, le $k y t$ qui est le potentiel de bonheur et de chance partagé par le nouveau né avec son lignage pour toute sa vie et enfin, le souffle que les humains ont en commun avec les animaux, la montagne et d'autres éléments de la nature. 
à chacun de rester dans une certaine mesure kazakh, kirghiz, ouzbek, tadjik, karakalpak, russe... La fameuse séparation entre citoyenneté et nationalité était visiblement très opératoire, puisqu'elle avait laissé fonctionner les référents culturels pré-soviétiques malgré l'oppression et la clandestinité.

Une des clefs de lecture de la situation contemporaine est la rupture introduite et voulue par les autorités depuis l'indépendance.

L'identité culturelle est une aspiration légitime, mais coupée de la nécessaire prise de conscience de la situation historique, elle est dangereuse. Ce n'est pas un concept instrumentalisable. D'abord, lorsqu'une collectivité commence à prendre conscience de son identité culturelle, il y a fort à parier que celle-ci est déjà irrémédiablement compromise. L'identité culturelle existe en soi dans les groupes vivants. Quand elle devient pour soi, elle est déjà le signe d'un repli face à une menace; elle risque de s'orienter vers l'enfermement, voire l'imposture (Latouche $2005: 17$ ).

Cette imposture signalée par Serge Latouche apparaît très pertinente dans le cas kazakh et ce, particulièrement en milieu urbain. Comme déjà signalé, la plupart des groupes sociaux intensément russifiés dans les villes ont perdu jusqu'à l'usage de leur langue maternelle. La ré-écriture de l'histoire kazakhe au regard de grandes figures telles que Gengis Khan et Timour, en gommant l'histoire récente, conduit à un artifice dévastateur. Un Kazakh qui ne parle pas kazakh doit aujourd'hui se justifier et se créer vaille que vaille une identité de Kazakh. La kazakhification entreprise à l'indépendance, à tous les échelons de la société, a marqué la revanche linguistique des campagnes sur les villes mais simultanément, elle a fait exploser l'organisation de la société traditionnelle, en propulsant les populations vers un monde inconnu.

Un exemple de changement dans les habitudes quotidiennes est celui de la consommation d'alcool. La période soviétique et sa politique du litre de vodka moins cher que le litre de lait, avait conduit à l'alcoolisme une majorité de ces populations. Depuis peu, la consommation baisse, et ce particulièrement lors des fêtes familiales ou des rites de passage tels que le mariage. Peut-on imputer cette diminution de la consommation d'alcool à un retour des pratiques en islam ? Je ne le pense pas ou, en tout cas, pas seulement. Le mythe du sain et du malsain, du bien vivre et du bien manger, qui est apparu il y a déjà quelques années en Occident, a rattrapé l'Asie centrale. Le marché des produits naturels, des vitamines et des compléments alimentaires est en pleine expansion dans ces régions. De même, le fitness, le jogging et autres entraînements corporels sont à la mode. Les salles de sport, caricatures du modèle américain, se multiplient aujourd'hui dans les grandes villes kazakhes. La diminution de la consommation d'alcool dépend ainsi d'un ensemble de facteurs où l'argument religieux n'a qu'un rôle limité.

Il est vrai, le sport - comme l'enseignement - pour tous représentait l'un des fleurons de l'ex-URSS ; chacun, à l'époque, avait le loisir de fréquenter gratuitement les installations sportives alors qu'aujourd'hui, il faut payer pour fréquenter les salles de fitness. Ce n'est donc pas le culte du sport que l'Occident a apporté, mais sa marchandisation, en même temps qu'un certain souci du corps propre à la société de consommation.

Cet exemple montre comment émerge petit à petit l'individu au cœur du communautaire : le prendre-soin-de-soi indépendamment des autres. Une référence jusqu'alors impensable dans un système où le complexe du mal, du malheur et de la maladie se gérait collectivement puisqu'il affectait la communauté tout entière et pas seulement l'individu. Loin de moi l'idée de faire l'apologie de l'alcool partagé qui se doit 
de couler à flot, mais force est de constater que ce changement apparemment insignifiant indique un virage radical dans les pratiques communautaires.

La perte de repères collectifs et le repli sur la famille nucléaire risquent d'atteindre en profondeur l'organisation sociale. Jusqu'alors, le cadet héritait de la yourte parentale et s'occupait de ses vieux parents. Les grands-parents recevaient le premier-né de l'aîné de leurs enfants, premier-né qui devenait ainsi leur dernier enfant et donc leur cadet. La fuite vers les villes et l'obligation de se trouver du travail en dehors de la succession familiale laissent apparaître un isolement dramatique des personnes âgées. Mourir seul et sans ressources, sans une belle-fille pour s'occuper du foyer, était jusqu'alors impensable ; aujourd'hui, c'est monnaie courante.

Ainsi l'impensable est-il en train de se réaliser, tout en restant impensé. Comment cette nouvelle société parviendra-t-elle à résoudre ces contradictions ? La société kazakhe va-t-elle adopter nos mouroirs pour ses anciens et nos crèches pour ses nouveaux-nés?

\section{Le nouveau monde}

La situation est pour le moins paradoxale. La pluralité des mondes organisés autour de l'axe vertical de l'arbre des mondes tend à disparaître au profit d'un monde au singulier, mais ce dernier se trouve mondialisé avant même d'avoir eu le temps de s'organiser.

Comme l'a écrit Mike Singleton :

Au-delà des nomades de l'histoire, dont certains, forcés et contraints d'errer, ne rêvaient que de pouvoir prendre racine, il y a le nomadisme : le perpetuum mobile libérateur, sans terminus a quo ni terminus ad quem bien définis et désirés, ni un retour-recours en arrière, ni un pari sur l'avenir, un simple aller de l'avant, mais qui n'a rien d'un tourner en rond, tout au plus un progresser en spirale qui contourne tous les culs-de-sac, l'essentiel étant de cheminer, encore et toujours pour éviter l'arrêt de mort qui frappe le sédentaire - même celui qui s'arrête dans le paradis post-développementaliste. (...) Une fois définitivement arrivé, le sédentaire sauvé n'a pas plus de raison de continuer à bouger à droite et à gauche sur Terre qu'au Ciel (Singleton 2004 : 199).

En se rappelant que les mondes kazakhs se superposaient les uns aux autres, chacun habité par des divinités et des figures d'esprits, mais aussi que le monde des humains se structurait d'après les points cardinaux alliant la gauche au nord et la droite au sud, en se remémorant encore que l'incarnation terrestre s'organisait en cycle à partir de l'ouest vers l'est, lieu de la naissance, jusqu'au retour de la mort à l'ouest, comment la société kazakhe contemporaine peut-elle gommer sa raison profonde de mouvement?

Les arguments politiques, religieux ou sociaux ne montrent-ils pas leurs limites dans ce type de situation? Depuis «le désenchantement du monde » et " l'occidentalisation du monde », nombre de penseurs et de courants se cramponnent à la raison instrumentale dont résulterait, comme l'écrit Jean-Pierre Dupuy, « le ravalement de tout être et de toute chose au statut de simple moyen au service d'une fin qui le dépasse » (Dupuy 2002 : 31). Je rejoins cet auteur lorsqu'il souligne l'originalité d'Yvan Illich dans sa critique du mode industriel de production :

Ce qui définit ce dernier, selon lui (Illich), ce ne sont pas les rapports de production, selon la caractérisation marxiste, ni même un certain type de rapport technique à la nature. $\mathrm{Au}$ fondement, on trouve la logique du détour. Cette logique elle-même a ses racines dans le religieux (Dupuy 2002 : 32). 
Dans le cas kazakh, il faut en effet quitter la représentation que l'homme kazakh se fait de lui-même, accepter le détour pour tenter de comprendre ce qui se joue aujourd'hui et qui semble se pérenniser. Il s'agit littéralement d'un changement de paradigme qui conduit une société nomade communautaire à devenir une société sédentaire individualisante.

Plus encore, nous assistons à la substitution, à la réalité perdue, de sa représentation exaltée tantôt générée par les acteurs eux-mêmes, tantôt instrumentalisée par le pouvoir. Le virtuel se substitue au réel et ce dans les fondements même de la nation : passage à une identité nationale, c'est-à-dire à une communauté imaginaire, à une identité religieuse musulmane et à l'appartenance à une communauté tout aussi imaginaire, celle de la grande communauté musulmane internationale. Cette société kazakhe semi-nomade s'est trouvée - et se trouve encore - confrontée aux mouvements fondamentaux de la modernité, à savoir : les transformations chroniques du réel en sa représentation et peu à peu à une inscription de leur(s) monde(s) dans un monde virtuel globalisé. 


\section{Références bibliographiques}

Apadurai, Arjun, 2001. Après le colonialisme, les conséquences culturelles de la globalisation. Paris, Payot.

Barthold, W, 1962. Four Studies on the History of Central Asia. Traduit du russe par V. et T. Minorsky, Leyde, E.-J. Brill, vol. I.

Bonte, Pierre et Michel Izard, 1991. Dictionnaire de l'ethnologie et de l'anthropologie. Paris, PUF.

Dupuy, Jean-Pierre, 2002. Pour un catastrophisme éclairé. Quand l'impossible est certain. Paris, Seuil.

Dor, Rémy, 1975. Contribution à l'étude des Kirghiz du Pamir afghan. Paris, Publications Orientalistes de France (Cahiers Turcica).

IANKovski, H., 2005. «Peremeni na iazikovoï karte kazakhstana i kirgizstana. Shodstva i razlitchiia » [Changements de la carte linguistique kazakhe et kirghize. Analogie et différences], in Shygys, Institut Vostokovedeniia im. R.B.Suleïmenova, Almaty, volume 2, pp. 80-89.

Krader, L., 1963. Social Organisation of the Mongol-Turkic pastoral Nomads. La Haye, Mouton (Uralic and Altaic series, 20).

Laruelle, Marlène et Sébastien Peyrouse, 2006. Asie centrale, la dérive autoritaire. Cinq républiques entre héritage soviétique, dictature et islam. Paris, Editions Autrement.

Latouche, Serge, 2005. L'occidentalisation du monde. Paris, La Découverte.

Mardanov, Kamalbaï, 2004. « Sovremiennie Kazakhi : pamiat nomadisma i petchat urbanisatsii » [Les Kazakhs contemporains : mémoire du nomadisme et empreinte de l'urbanisation], in Urbanizatsiia i Nomadizm $v$ Tsentralnoï Azii : Istoriia i problemi, Materiali mezhdounarodnoï konferentsii, [Actes de la conférence internationale : Urbanisation et Nomadisme en Asie centrale : histoire et enjeux], Almaty, pp. 431-441.

Nysanbaeyev, Abdumalik, 2004. Kazakhstan, Cultural Inheritance and Social Transformation. Washington, Kazakh Philosophical Studies, I, The Council for Research in Values and Philosophy.

Pelliot, Paul, 1950. Notes sur l'histoire de la Horde d'Or. Paris, Adrien-Maisonneuve.

Rahnema, Majid, 2003. Quand la misère chasse la pauvreté. Paris, Fayard-Actes Sud.

Roux, Jean-Paul, 1984. La religion des Turcs et des Mongols, Paris, Payot.

Singleton, Mike, 2004. Critique de l'ethnocentrisme. Du missionnaire anthropophage à l'anthropologue postdéveloppementiste. Paris, Paragon.

SLOTERDijk, Peter, 2002. Bulles, Sphères 1. Paris, Fayard.

Valikhanov, Tchokhan, 1985 ( $1^{\mathrm{e}}$ édition 1834-1860). Sobranie sotchinenï v pjati tomakh, [Euvres complètes en cinq tomes], Almaty.

VuilLEMENOT, Anne-Marie,

2000. «Au Kazakhstan les yourtes se vident depuis l'indépendance ». In Firouzeh Nahavandi (dir.). Globalisation et néo-Libéralisme dans le Tiers monde, pp. 155-169. Paris, L'Harmattan (Bibliothèque du développement).

2002. «Défaire le développement, refaire les mondes kazakhs ». In : La ligne d'horizon, Défaire le développement refaire le Monde, pp. 240-248. Paris, Paragon.

2006. «L'islam populaire kazakh et ouzbek : religion, guérison, nation », in : Recherches Sociologiques et anthropologiques (Islam : entre local et global), vol. XXXVII (2), pp. 63-78. Louvain-la-Neuve, Université Catholique de Louvain, Unité d'anthropologie et de sociologie. 